Archives

$10 \mid 1993$

Le Centre de recherches historiques de 1949 à 1975

\title{
La première structure organisationnelle :
}

\section{7-1965}

\section{Q OpenEdition}

\section{Journals}

Édition électronique

URL : http://journals.openedition.org/ccrh/2789

DOI : $10.4000 /$ ccrh. 2789

ISSN : $1760-7906$

Éditeur

Centre de recherches historiques - EHESS

Édition imprimée

Date de publication : 15 avril 1993

ISSN : 0990-9141

Référence électronique

"La première structure organisationnelle : 1957-1965 », Les Cahiers du Centre de Recherches

Historiques [En ligne], 10 | 1993, mis en ligne le 16 mars 2009, consulté le 04 mai 2019. URL : http:// journals.openedition.org/ccrh/2789; DOI : 10.4000/ccrh.2789

Ce document a été généré automatiquement le 4 mai 2019.

Article L.111-1 du Code de la propriété intellectuelle. 


\section{La première structure organisationnelle : 1957-1965}

1 Une série d'événements transformèrent profondément les conditions d'existence du CRH entre 1956 et 1958. La mort de Lucien Febvre en 1956 élargit le champ d'action du directeur du CRH : F. Braudel devenait directeur de la VI Section et l'homme fort du comité directeur des Annales ESC. Cette unité de direction rapprochait à terme le réseau des Annales ESC à celui de la VI ${ }^{e}$ Section en donnant aux activités scientifiques du CRH un écho plus large. La création de 11 postes de directeurs et sous-directeurs d'études et de 7 postes de chefs de travaux - un poste de début de carrière manquait jusque-là à la $\mathrm{VI}^{\mathrm{e}}$ Section et posait l'alternative de promouvoir très tôt un jeune chercheur à un poste de directeur d'études (le cas de R. Romano) ou de l'aider à poursuivre sa carrière en dehors de la VI ${ }^{e}$ Section - entre 1956 et 1958, fut le point de départ d'une première phase de croissance accélérée qui culmina entre 1958 et 1961 avec la nomination de 55 chefs de travaux et de 18 directeurs et sous-directeurs d'études ${ }^{1}$. La division d'histoire et le CRH en profitèrent avec la nomination de 4 sous-directeurs dont deux au CRH (Ozanam et Glénisson) et celle de 16 chefs de travaux qui créaient un véritable corps de chercheurs au CRH.

2 Ces changements morphologiques influèrent sur les structures d'organisation du Centre. D'abord son directeur, absorbé par ses activités de président de la $\mathrm{VI}^{\mathrm{e}}$ Section et de la Maison des Sciences de l'Homme, commença à déléguer des pouvoirs au secrétaire général : D. Ozanam de 1957 à 1961, R. Philippe de 1961 à 1963 et R. Romano de 1963 à 1965 se virent confrontés, l'un après l'autre, au problème de gérer un centre qui était toujours à la recherche de modèles organisationnels capables de structurer les initiatives scientifiques multiples qui résultaient de la croissance de l'institution et du rayonnement intellectuel de «l'école historique française ».

3 Les nouveaux chercheurs qui rejoignirent la $\mathrm{VI}^{\mathrm{e}}$ Section pendant ces années trouvèrent un Centre de recherches historiques qui s'était doté de structures internes plus stables. Le rapport présenté par son secrétaire général, Didier Ozanam fin 1957 en esquissa les lignes directrices : pour faire un pas en avant vers un véritable centre de recherches collectives, le CRH ne devait pas « rester un CNRS (sciences humaines) qui met, sur leur demande, des moyens financiers et matériels à la disposition des historiens ", il devait constituer le 
prototype du laboratoire de recherche historique doté d'un programme fixe, d'un personnel stable et intégré organiquement au Centre, partageant ses locaux et disposant d'un budget fixe ${ }^{2}$. Ozanam insista en outre sur la nécessité de définir des buts de travail collectifs concrets: publication de textes, de répertoires et d'inventaires, enquêtes bibliographiques.

Tableau 7. Groupes de recherches au CRH 1957-1962

\begin{tabular}{|c|c|}
\hline \multicolumn{2}{|c|}{ 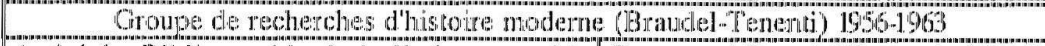 } \\
\hline 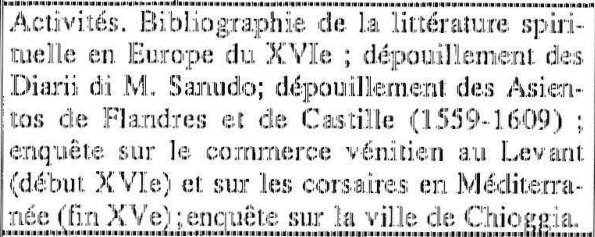 & 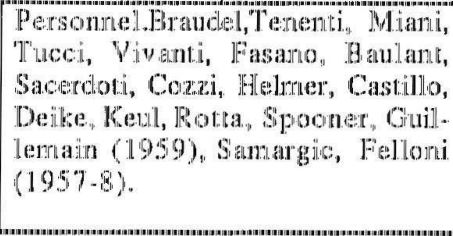 \\
\hline \multicolumn{2}{|c|}{ 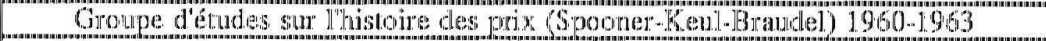 } \\
\hline 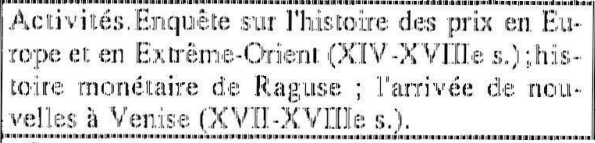 & $\begin{array}{l}\text { Personel, Bpoomer, Braudel, Kevil, } \\
\text { Andrews, Baulant.. }\end{array}$ \\
\hline \multicolumn{2}{|c|}{ 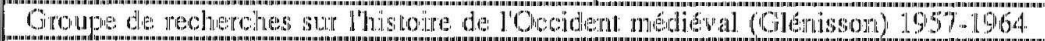 } \\
\hline 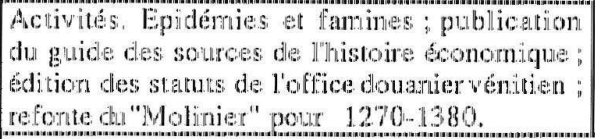 & $\begin{array}{l}\text { Persomel, Giénissom, Carpentier } \\
\text { Gasmault, Bigounes,Somay, Payen. }\end{array}$ \\
\hline \multicolumn{2}{|c|}{ 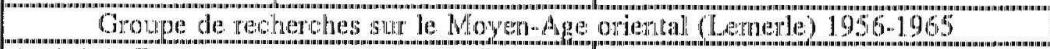 } \\
\hline 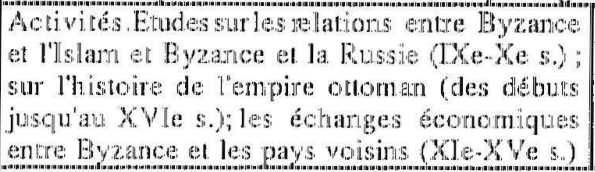 & 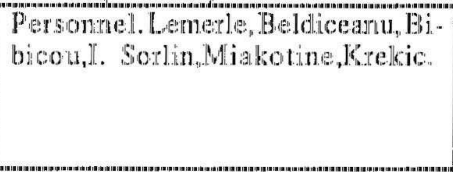 \\
\hline \multicolumn{2}{|c|}{ Growpe de recherches sur l'hisoue des entreprises et des techniques (Gille) 1957.63} \\
\hline 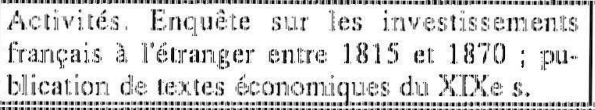 & $\begin{array}{l}\text { Personinel.Gille Qulitichini., Vou al, } \\
\text { Grenat. }\end{array}$ \\
\hline
\end{tabular}

Suivant cette orientation, le CRH fut organisé à partir de 1957-58 en quatre groupes de recherche qui formaient des équipes de 3 à 8 chercheurs dirigées par un directeur d'études et qui poursuivaient leurs enquêtes dans le cadre d'un programme de recherche bien défini ${ }^{3}$.

Le budget de 1959 donne un indice du poids relatif de chaque groupe de recherche, un bon tiers des moyens étaient alloués à ceux de F. Braudel, les groupes byzantinistes et médiévistes recevaient 28,9 et $22,7 \%$ respectivement, et l'histoire des entreprises seulement un peu plus de $15 \%$.

6 Quel est le bilan de cette réorganisation qui voulait être un pas vers la mise en place d'un véritable laboratoire de recherche historique? L'irrésistible expansion des années suivantes a produit un effet d'émulation. En 1962, une bonne douzaine de groupes existaient au CRH sans qu'un dynamisme de recherche fût à l'origine de toutes ces créations mais la logique de la hiérarchie universitaire qui incitait à la naissance de services de secrétariat et d'assistances de recherche autour de chaque direction d'études. Comme le CRH continuait en même temps de fonctionner comme distributeur secondaire de subventions et d'aides à des chercheurs ou groupes de recherche extérieurs, une conséquence imprévue de cette réorganisation était le risque d'éclatement du Centre et un émiettement des ressources. En 1963, en dehors des groupes de recherches et des 
grandes enquêtes, le CRH subventionna les projets de recherche individuelle de 10 autres chercheurs par un ou deux postes de vacation.

7 Par une autre tentative de recentrage et de réorganisation, une nouvelle direction essaya de corriger cette évolution. En 1963, R. Romano prépara une autre phase du CRH en supprimant «le plus grand nombre possible de petits centres et groupes, qui s'étaient constitués à l'intérieur du Centre proprement dit, pour organiser plutôt le travail sous forme d'enquêtes aux termes fixés avec suffisamment de précision ${ }^{5}$ ». À la fin de cette réorganisation en 1965, tous les sous-centres avaient disparus ou étaient en passe d'être dissous.

8 Le premier groupe constitué en 1957 était en fait le réseau le plus ancien du CRH et ses nouveaux ou anciens membres poursuivaient les recherches sur les problèmes économiques et sociaux $\mathrm{du} \mathrm{XVI}^{\mathrm{e}}$ et $\mathrm{XVII}{ }^{\mathrm{e}}$ siècles dans le monde méditerranéen. Les deux groupes médiévistes et byzantins marquent une étape d'élargissement thématique importante. Bien que l'histoire économique et sociale du Moyen Âge fût présente dans le programme d'enseignement de la $\mathrm{VI}^{\mathrm{e}}$ Section dès l'origine (par l'enseignement de M. Lombard), le CRH n'avait pris aucune initiative de recherche ou de publication. Dans le cas du groupe de Lemerle, le CRH intégra tout un programme de travail ${ }^{6}$ et un groupe de chercheurs sans lier son directeur à la $\mathrm{VI}^{\mathrm{e}}$ Section. P. Lemerle, directeur d'études à la IV ${ }^{\mathrm{e}}$ Section depuis 1947, fonda en 1959 le Centre de recherches d'histoire et de civilisation byzantines, préférant l'autonomie institutionnelle à l'intégration dans le Centre de recherches historiques. Il en résulta que le $\mathrm{CRH}$ fonctionnait comme centre financier et figurait comme structure formelle d'accueil de crédits, mais le groupe et son travail restaient à l'écart des autres groupes du CRH. Après la mise en route de son propre centre, Lemerle y transféra la plus grande partie de son équipe, ne laissant au CRH que Mme Antoniadis qui, tout au long de sa carrière au CRH, a poursuivi des recherches sur l'histoire économique et sociale de Byzance et (plus tard) sur la Grèce moderne. Les recherches byzantines ne regagnèrent plus de terrain que dans les années 1970 avec l'élection de M. Guillou comme directeur d'études.

9 Les groupes de travail de Glénisson et de Lemerle se consacraient à des travaux de documentation et d'édition qui cherchaient à reprendre la tradition des éditions scientifiques du $19^{\mathrm{e}}$ siècle. À bien des égards, l'Institut de recherche et d'histoire des textes du CNRS restait le modèle implicite de ces groupes. Avec la dissolution du groupe de recherche sur l'histoire du Moyen âge occidental à la suite de la nomination de Glénisson à la direction de l'IRHT en 1965, ce type d'activités érudites a perdu toute importance au CRH et, avec lui, tout le modèle de laboratoire historique orienté vers des activités de documentation ou de préparation à la recherche.

10 Plus proche des préoccupations thématiques dominantes au Centre était l'équipe de B. Gille qui élargissait la recherche d'histoire économique du CRH au XIX siècle et à l'époque du capitalisme industriel.

11 Mais à côté des activités de ces équipes plus au moins autonomes et stables, le CRH est devenu un champ d'expérimentation pour toutes sortes d'initiatives et d'idées que le groupe annaliste rassemblé autour de F. Braudel développa pendant ces années qui consacrèrent en quelque sorte son succès intellectuel à l'intérieur de l'historiographie française $e^{7}$.

12 Après le démarrage des recherches sur l'histoire économique, le CRH traversa une phase de redéfinition de ses objectifs qui, à l'époque, étaient pratiquement ceux du groupe 
annaliste autour de F. Braudel. Après ses prises de position théorique des années 1958 à 1960, il plaida en faveur d'une accélération des enquêtes et d'une ouverture de nouveaux chantiers. La reprise des comptes-rendus d'enquêtes dans les Annales ESC en 1961 marquait la nouvelle direction :

L'avantage des enquêtes de Marc Bloch et de Lucien Febvre a même résidé, pensons-nous, dans leur brièveté : elles ont, à un moment donné, signalé des intérêts, des recherches possibles et ainsi atteints leurs buts exacts ; [...] la logique d'une enquête conçue à dessein comme l'occupation rapide d'un vaste terrain qu'il s'agit de découvrir, de mesurer, de révéler dans son ampleur, avant d'avoir la prétention d'en exploiter ou d'en dominer l'espace ${ }^{8}$.

Ce terrain, c'était l'histoire sociale conçue à la fois comme analyse de la vie matérielle, des sociétés et des civilisations et Braudel critiqua à plusieurs reprises la tendance des historiens à choisir des sujets trop étroits et sans envergure théorique ${ }^{9}$. Il ne cessa d'inciter les historiens à s'inspirer des modèles économiques sur les cycles ou sur les facteurs de croissance, des modèles démographiques et des théories sociologiques, et il reformulait l'ambition des Annales d'histoire économique et sociale d'avant 1939 de faire de leur discipline une "science sociale actuelle", de pratiquer "une histoire qui est intelligence de l'actuel $»^{10}$.

Les traces des discussions internes sur la politique scientifique et les témoignages oraux nous présentent un Centre de recherches historiques que ses membres ont l'intention de transformer en centre d'une " histoire d'avant-garde », d'une " histoire révolutionnaire » qui transférerait les «méthodes traditionnelles de recherche et de critique dans des régions jusqu'ici mal connues $»^{11}$. L'ambition était de se démarquer d'une histoire traditionnelle définie laconiquement par Braudel, dans cette même réunion, comme « celle qui se faisait avant $1939^{12}$ ».

Le CRH devenait le lieu d'expérimentation de cette ambition qui, faute de critères méthodologiques ou théoriques clairs pour choisir dans une masse infinie de sujets de recherche, a produit une politique volontariste. Il en résulta des choix tout pragmatiques qui souvent ne faisaient que reprendre les idées suggérées par d'autres, comme, par exemple, l'étude de la vie matérielle par des fouilles de villages désertés - une idée que F. Braudel rapportait d'un voyage en Pologne- ou l'enquête sur les psychologies collectives - où l'on voit se dessiner le projet de recherches sur les pèlerinages dirigés par Dupront au CRH à partir de 1964. À côté de ces recherches qui vont être réalisées au cours des années 1960, il y a toute une série de propositions qui sont restées lettre morte ou affaire personnelle d'un chercheur du CRH, telles la proposition de s'engager sur le terrain de la génétique historique, l'idée d'une enquête internationale sur une grande crise historique, celle des années 1619-1622, le projet de recherches et de publications sur l'Amérique Latine lancé en 1963 par R. Romano ${ }^{13}$ ou d'une enquête sur les prix en Inde et en Chine - ce dernier projet soulevant toute de suite le problème des relations entre le $\mathrm{CRH}$ et les "Aires Culturelles ${ }^{14}$ ». Un exemple typique des ambiguïtés liées à une telle situation sont les efforts plus ou moins dispersés de mettre en chantier des recherches d'histoire contemporaine. Les idées venaient d'un peu partout: Labrousse proposa des recherches sur la Commune (le dépouillement des dossiers du Conseil de guerre par des procédés mécanographiques), R. Fisher pourvut à l'existence éphémère d'un centre de recherches sur l'histoire du marxisme dans la division des aires culturelles en 1960 et 1961, J.-P. Peter fut encouragé par F. Braudel à explorer l'histoire du scandale au XIX ${ }^{\mathrm{e}}$ siècle et la période du Front populaire ${ }^{15}$. Toutes ces initiatives sont nées au début des années 1960, elles ont été poursuivies quelques mois et même quelques années mais elles 
n'ont jamais trouvé le soutien d'un programme à long terme, de sorte que l'inertie des décisions antérieures pour une histoire économique des $\mathrm{XVI}^{\mathrm{e}}$ et $\mathrm{XVII}^{\mathrm{e}}$ siècles ou celles, simultanées, pour des enquêtes lourdes axées sur des sujets plus proches des préférences thématiques personnelles du directeur du CRH, finit par laisser se refermer ces tentatives d'ouverture ${ }^{16}$.

Un autre exemple d'enquête abandonnée de cette époque nous fait voir les barrières concrètes dressées contre la pratique de recherche interdisciplinaire pourtant officiellement prônée: Le projet d'une telle enquête interdisciplinaire associant historiens du CRH (sous la direction de R. Romano) et économistes de l'ISEA (des équipes de F. Perroux et J. Marczewski) sur la reconstruction du revenu national français de 1726 à nos jours ${ }^{17}$ répondait à la fois aux progrès réalisés dans les recherches d'histoire économique à base statistique ou quantitative et à l'intérêt renouvelé des économistes pour l'histoire. Mais les équipes du " groupe d'études sur la croissance économique de la France ", placées officiellement sous le contrôle de F. Perroux, E. Labrousse et F. Braudel, n'ont pas réussi à publier de résultats communs. La confrontation de l'approche de la « New Economic History » et de la tradition annaliste s'est montrée peu fructueuse. Sous la direction de Jean Marczewski, l'ISEA a publié de 1961 à 1969 les résultats de ses reconstructions quantitatives du produit national et de la croissance économique de la France de 1726 à nos jours ${ }^{18}$. Du côté historique, l'enquête poursuivie en 1960, 1961 et reprise en 1963 et 1964 a finalement été abandonnée sans présenter de résultats. Même la discussion théorique et méthodologique tourna court et la rupture des historiens de l'économie annalistes avec leurs collègues de la «New Economic History» devint complète ${ }^{19}$.

17 Parmi ces investigations tous azimuts, trois projets ont remporté la palme au cours des années 1961 et 1962 : les recherches sur l'histoire alimentaire, l'enquête sur les villages désertés et celle sur le livre et la société dans la France du XVIII ${ }^{e}$ siècle. Les deux premières enquêtes pouvaient donner des éléments de réponses aux nouvelles recherches de F. Braudel et son groupe sur l'histoire de la vie matérielle. Les documents touchant l'habitation, les costumes, les conditions techniques, l'alimentation et les régimes alimentaires satisfaisaient le goût du concret qui a caractérisé toutes les recherches d'histoire économique et sociale inspirées et initiés par Braudel et le CRH. Ces sujets permettaient d'ouvrir des chantiers contigus aux anciens sur les prix et le commerce, et ils avaient l'avantage d'occuper le rez-de-chaussée dans l'architecture des domaines historiques que F. Braudel avait tout récemment exposée. Mais il ne faut pas oublier que les suggestions venaient des historiens et archéologues polonais qui étaient en ces années les interlocuteurs étrangers privilégiés de leurs collègues du CRH.

18 Dans le cas des deux enquêtes sur les villages désertés et sur le livre et la société, Braudel servait, avec ses intérêts intellectuels, ses ambitions scientifiques nationales et internationales. Son Centre commençant à disposer d'un équipement en personnel et financier suffisant, il offrit, dans les institutions historiques internationales, ses services aux projets d'enquêtes internationales ce qui lui permettait de conforter sa renommée et celle de toute l'école historique française. 


\begin{tabular}{|c|c|}
\hline $\begin{array}{l}\text { Fouilles âSt Jean le Froid, Montagut er Dracy ; } \\
\text { recherches sur les villages désertés en France, } \\
\text { en Italie et en Grèce. }\end{array}$ & 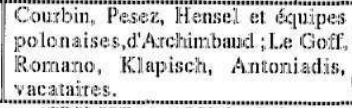 \\
\hline \multicolumn{2}{|c|}{ Le tevenu national francais de 1726 a nos jours (CRH -ISEA) (1958 $1963 / 4)$} \\
\hline 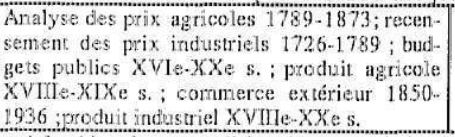 & $\begin{array}{l}\text { Pertoux, Marczewsiki, Braudel, La- } \\
\text { brousse, Romarno, Piatien, Marco- } \\
\text { vitch, Antorniadis, Weiller, Tou- } \\
\text { tain, } 15 \text { vacataires en } 1960 \text {. }\end{array}$ \\
\hline \multicolumn{2}{|c|}{$\begin{array}{l}\text { Atlas hissorigue mondial plantes cultivées animaux domestioues (1961-1968) } \\
\text { |Keul Hérnardinouer, Randles La- }\end{array}$} \\
\hline & $\begin{array}{l}\text { Keul, Hérnardinquer, Randles, La- } \\
\text { boratogire de cartographie. }\end{array}$ \\
\hline \multicolumn{2}{|c|}{ Byzance er la méditerranée aux XIle et XIle siecles (9561-1965) } \\
\hline $\begin{array}{l}\text { Waffaizes genois et vénitiens; commu- } \\
\text { ves rnarchands occidentaux äByzance }\end{array}$ & $\begin{array}{l}\text { Antoniad is, Triantaphyllid } \\
\text { bois, Klapisch }\end{array}$ \\
\hline \multicolumn{2}{|c|}{ Livre es seciêe dans la France du XVIIIe siacle (1962.1965) } \\
\hline $\begin{array}{l}\text { Liste exhaustive des livres qui ont sollicité } \\
\text { l'autorisation royale; études sur les livres de } \\
\text { colportage, comptes-rendus dans le Journal } \\
\text { des Savants et les Mémoires de Trévoux; le } \\
\text { personnel des acadérnies de province; les re. } \\
\text { gistres de perrnission d'imprimer. }\end{array}$ & $\begin{array}{l}\text { Fure, Ehrard (Clemont- Ferrand) } \\
\text { Roger (Poitiers), Roche (ENS St- } \\
\text { Cloud), Bolleme, } 5 \text { vacataires. }\end{array}$ \\
\hline \multicolumn{2}{|c|}{ Le mouvernent du profit en France au XOXe siecle $(1962-1965)$} \\
\hline $\begin{array}{l}\text { Depouillement de journaux et publications } \\
\text { économiques français du XTXe s. et des dos- } \\
\text { siers des Axchives nationales surt les bançues, } \\
\text { les mines, I'industrie sidérurgique. }\end{array}$ & $\begin{array}{l}\text { Bouvier, Furet, Gillet, Levain } \\
\text { (CNRS), 1 vacataire. }\end{array}$ \\
\hline \multicolumn{2}{|c|}{ Les pélerinages dans liEurope moderne (1964-1976?) } \\
\hline $\begin{array}{l}\text { Carte des pelerinages actuels en France ; fiche } \\
\text { documentaire sur chaque pélerinage; collec- } \\
\text { tion de documents concernant les pélerinages } \\
\text { européens. }\end{array}$ & $\begin{array}{l}\text { Dupront, Ailleret, Aurigermura va:- } \\
\text { cataires. }\end{array}$ \\
\hline \multicolumn{2}{|c|}{ 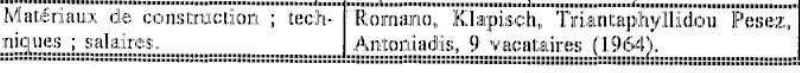 } \\
\hline
\end{tabular}

Ont donné lieu à publications : Villages désertés et histoire économique, XI-XVIII siècles, 1965 ; Bollème, Ehrard, Furet, Roche, Roger, Livre et société dans la France du XVIII siècle, 1965 : Bouvier, Furet, Gillet, Mouvement du profit en France au XIXe siècle, 1965. La dernière enquête a été réorganisée sous la direction de Le Roy Ladurie après 1965 et s'est intitulée « Maison et bâtiment dans l'histoire économique » (1965-1970). Pour un résultat partiel de l'enquête originale, cf. la thèse de KlapischZuber, Le marbre toscan du XII au Xv| $\mathrm{e}$.

L'enquête sur les villages désertés avait été lancée par le Comité d'histoire économique lors du congrès de Stockholm, soutenue par le Comité directeur de la section du CNRS et devait être présentée au troisième Congrès d'histoire économique à Munich en 1965. En acceptant le rôle de coordinateur de l'enquête internationale, les historiens du CRH pouvaient profiter de l'occasion pour rattraper le retard des recherches nationales dans le domaine de l'archéologie médiévale et sur le terrain spécial des villages désertés. L'effort de travail collectif mobilisé à cette occasion était sans commune mesure avec l'engagement dans les recherches collectives antérieures. L'enquête sur le livre et la société est issue d'une proposition de F. Braudel au Comité international des Sciences Historiques et ses résultats furent présentés au Congrès international à Vienne en 1965. Dans ce cas, le CRH assurait le patronage d'une recherche qui reposait largement sur la collaboration de chercheurs extérieurs, tel Ehrard, professeur à l'université de ClermontFerrand, de Roger, alors professeur à l'université de Poitiers ou D. Roche, assistant à l'ENS de St. Cloud. Du côté du CRH, F. Furet et G. Bollème s'occupaient de la coordination de l'équipe parisienne sous la direction scientifique d'A. Dupront.

Avec cette enquête, le CRH faisait ses premiers pas dans le domaine de l'histoire culturelle en transposant les méthodes d'histoire sérielle à un terrain jusque-là négligé par lui. En fait, les explorations de L. Febvre, de R. Mandrou ou de G. Duby - tous les trois plus ou moins distants du Centre pendant les années 1950 - sur le terrain d'une histoire des 
mentalités ou d'une histoire de psychologie collective n'avaient trouvé aucun écho dans les enquêtes du CRH. La création des nouveaux postes permettait à la direction de la $\mathrm{VI}^{\mathrm{e}}$ Section d'intégrer l'histoire religieuse, l'histoire sociale et culturelle aux programmes d'enseignement et de recherche de la maison. Comme nous l'avons vu, dans les années 1958 à 1963, la création d'un bon nombre de nouvelles directions d'études, suivant fréquemment la vogue sociologisante, s'orientaient vers une histoire sociale des phénomènes culturels. En histoire du Moyen-Âge, les cours de J. Le Goff marquaient dès 1960 un élargissement de la problématique classique d'histoire économique qu'a représentée à la $\mathrm{VI}^{\mathrm{e}}$ Section l'enseignement de $\mathrm{M}$. Lombard ${ }^{20}$. Sa direction d'études «Histoire et sociologie de l'occident médiéval» en 1962 exprime bien les nouvelles tendances. Le colloque international de 1962 sur « Hérésies et sociétés », organisé par le CRH (surtout par J. Le Goff) donnait très vite un écho international à cette réorientation. L'histoire religieuse $\mathrm{du} \mathrm{XVI}^{\mathrm{e}}$ siècle était le thème central des cours que J. Delumeau donnait à partir de 1963. Des directeurs ou sous-directeurs nouveaux, comme P. Jeannin et A. Tenenti mettaient leur enseignement sous le signe d'une histoire sociale élargie. Par contre, d'autres protagonistes d'une approche nouvelle des représentations collectives dans l'histoire comme J.-P. Vernant, I. Meyerson ou A. Dupront, enseignaient pendant ces années dans la division de sociologie et de psychologie de la $\mathrm{VI}^{\mathrm{e}}$ Section - détail qui sans doute traduit l'inertie des orientations économiques.

21 C'est surtout Alphonse Dupront qui a marqué les premières recherches d'histoire culturelle au $\mathrm{CRH}^{21}$. Il donnait aux premières tentatives d'une approche quantitative des phénomènes culturels une justification méthodologique et un but ambitieux: en interprétant le sériel, le fait massif comme trace des couches profondes des représentations mentales collectives, il légitimait une approche qui rompait par ses méthodes avec la bienséance de l'histoire des idées en traçant un horizon large d'explications nouvelles qu'une psychologie historique des profondeurs devait fournir; en insistant sur le poids des langages comme transformations de besoins profonds, de pulsions d'« une âme collective », il ouvrait en outre la voie à une coopération avec la linguistique qui était en train de devenir la science humaine pilote de l'époque. Bien que ses concepts et son langage fussent loin de l'esprit des Annales des années 1930 à 1950, l'architecture historiographique d'A. Dupront, qui cherchait des profondeurs mentales collectives au-dessous des surfaces culturelles et politiques, s'approchait du modèle braudelien dominant au CRH. Les deux approches privilégiaient les recherches «sur de larges espaces de temps ${ }^{22}$ » et insistaient sur un travail de description historique comme première étape irremplaçable de chaque modélisation générale.

Les années 1957 à 1965 figurent dans l'histoire du CRH comme une phase de transition caractérisée par la coexistence de tendances contradictoires, d'orientations divergentes et un taux élevé d'expérimentations et d'improvisations personnelles et thématiques : transition vers un modèle institutionnel plus stable avec l'arrivée des premiers chercheurs permanents attachés au $\mathrm{CRH}$; tendances contradictoires en ce qui concerne les modèles de laboratoire historique à suivre. En ce qui concerne ces dernières, deux tendances coexistaient, l'une qui conduisait à un centre scientifique de documentation, d'édition de sources, de bibliographies et d'autres outils et matériaux de base pour la recherche historique et à des enquêtes exigeant des investissements lourds en termes de personnel et de temps; l'autre qui allait dans le sens d'un centre d'expérimentation pionnier à la fois au niveau des méthodes et des terrains de recherche, ce qui exigerait une mobilité accrue des ressources intellectuelles et matérielles. Les ambitions et les 
conceptions de F. Braudel pendant ces années allaient certainement dans ce sens, mais l'orientation pragmatique que nous avons retrouvée au niveau des choix d'enquêtes et de sujets de recherche renvoie à l'inertie croissante d'une institution qui commençait à gérer son renom et ses traditions.

\section{NOTES}

1. Liste dans B. Mazon, op. cit., p. 141 et 142.

2. Rapport pour la réunion du CRH du 19 déc. 1957, op. cit. p. 6-7

3. "Ces groupes ont été constitués pour tenter une expérience de recherche coordonnée et organisée, transposant en quelque sorte dans le domaine des sciences humaines - et en les adaptant - certaines des méthodes en usage dans les laboratoires scientifiques. [...] Il est nécessaire d'insister sur le caractère nouveau de ces groupes de recherches: disposant d'un minimum de chercheurs permanents et de moyens de travail suffisants, ils permettront la formation d'équipes de travail dont les membres seront habitués à œuvrer ensemble ou en liaison étroite.» («Note sur les groupes de recherches». Budget primitif des recettes et des

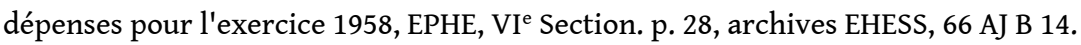

4. Budget de la VIe Section EPHE, archives EHESS 66 AJ B 23. Calculs effectués à partir des chiffres absolus de dépenses en matériel et en personnel.

5. Lettre de R. Romano à F. Braudel du 12 mars 1964, archives EHESS,. fonds Velay.

6. Paul Lemerle, «Les études d'histoire de Byzance et du Moyen Âge oriental », Annales ESC, 1955, p. 543-546

7. J. Glénisson a fait un commentaire très nuancé sur le nouveau consensus en faveur de « l'école des Annales" au début des années 1960 dans "L'historiographie française contemporaine: tendances et réalisations", in Comité Français des Sciences Historiques, Vingt-cinq ans de recherche historique en France 1940-1965, op. cit. p. IX-LXIV, voir surtout p. LXIII.

8. F. Braudel, Annales ESC, 1961, p. 41 et p. 546

9. Cf. son compte rendu peu enthousiaste de la thèse de P. Goubert, Annales ESC, 1963, p. 767-778.

10. Braudel, Annales ESC, 1961, p. 42.

11. Les termes sont de F. Braudel et de R. Romano, énoncés lors d'une réunion du CRH consacrée aux travaux poursuivis par le centre, le 24 juin 1960. (Procès verbal, archives EHESS, fonds Velay). L'investissement affectif et l'engagement personnel dans ces travaux collectifs «à la pointe » de la recherche historique ont été évoqués par tous les chercheurs de l'époque que j'ai pu interviewer.

12. Ibid., p. 3.

13. Projet américain (plan de démarrage), VI ${ }^{\mathrm{e}}$ Section de l'EPHE, budget 1963, archives EHESS, 66 AJ B 14.

14. Ibid., p. 4 et 5.

15. cf. Article dans les Annales et le rapport d'activités 1962-63 de Peter.

16. Les rapports d'activités de J.-P. Peter formulent clairement le dilemme : "J'estime qu'il n'y a aucun moyen de faire démarrer les études d'histoire du présent, hors de la construction d'une équipe, munie de moyens matériels suffisants et libre de préjugés, dirigée par un historien déjà formé et averti. Toute recherche portant sur l'histoire proche ne peut aboutir que dans un esprit 
résolument interdisciplinaire. C'est dire assez qu'à terme, l'historien cesse d'être historien. » Rapport d'activités 1963, archives EHESS, fonds Velay.

17. «Première rédaction d'un projet pour l'enquête en commun de l'ISEA et du CRH sur la croissance et le revenu national et le profit en France au $18^{\mathrm{e}}$ et au $19^{\mathrm{e}}$ siècle ", sans date, archives EHESS, fonds Velay; R. Mandrou, «Pour une enquête historique : le revenu national français de 1726 à nos jours ", Annales ESC, 1960, p. 752-758.

18. Cf. Jean Marczewski, «L'histoire quantitative de l'économie française », 10 vol., Cahiers de l'ISEA, Paris, 1961-1969. Du même, « Histoire quantitative - buts et méthodes ", ibid., cahiers nº 115, juin 1961, p. III-LIV.

19. P. Chaunu, «Histoire quantitative ou histoire sérielle", Cahiers Vilfredo Pareto, 1964, p. 165-175 ; J. Marczewski, «Buts et méthodes de l'histoire quantitative», ibid., p. 125-164 et "quelques observations sur l'article de M. Chaunu», ibid., p.177-180; P. Vilar, "Pour une meilleure compréhension entre économistes et historiens. "Histoire quantitative" ou "économétrie rétrospective"?", Revue historique, CCXXXIII (1965), p. 293-312; D. Richet, "Croissance et blocages en France du XV ${ }^{\mathrm{e}}$ au XVIII siècle », Annales ESC, 1968, p. 759-787 ; et le commentaire de Robert Forster, "The Achievements of the Annales School », Journal of Economic History, Vol. XXXVII (1978), p. 67 et sv.

20. Cf. ses cours sur «Idées religieuses et groupes sociaux en Occident du XI ${ }^{\mathrm{e}}$ au XIV ${ }^{\mathrm{e}}$ siècle » en 1960-61 et 1961-62, et sur «les peurs médiévales. Économies de subsistance et inquiétudes mentales » en 1962/63-1964/>65.

21. Cf. surtout ses réflexions méthodologiques et ses programmes de recherches : « Problèmes et méthodes d'une histoire de la psychologie collective" in Comité International des sciences historiques, XIe Congrès International des Sciences Historiques à Stockholm du 21 au 28/8/1960, Résumés des communications, Göteborg-Stockholm-Uppsala, 1960, p. 26-28; «De l'acculturation ", Comité International des Sciences Historiques, XII ${ }^{e}$ Congrès International des Sciences Historiques à Vienne du 29/8/1965 au 5/9/1965, Rapports 1 : Grands thèmes,. Vienne, 1965, p. 7-36; "Livre et culture dans la Société Française du XVIII siècle: Réflexions sur une enquête ", Livre et société dans la France du XVIII siècle, Paris-la Haye, 1965, p. 185-238.

22. A. Dupront, « Problèmes et méthodes ", op. cit., p. 27. 